\title{
Non-Fourier effect and inertia effect analysis of a strip with an induced crack under thermal shock loading
}

\author{
Wei Li ${ }^{\mathrm{a}, \mathrm{b}}$, Fan Song ${ }^{\mathrm{a}, *}$, Jia Li $^{\mathrm{b}}$, Radhi Abdelmoula ${ }^{\mathrm{b}}$, Chiping Jiang ${ }^{\mathrm{c}}$ \\ a State Key Laboratory of Nonlinear Mechanics, Institute of Mechanics, Chinese Academy of Sciences, Beijing 100190, China \\ b LSPM, CNRS UPR 3407 University Paris XIII, 99 Avenue Jean-Baptiste Clement, Villetaneuse 93430, France \\ ${ }^{\mathrm{c}}$ School of Aeronautic Science and Engineering, Beihang University, Beijing 100191, China
}

\section{A R T I C L E I N F O}

\section{Article history:}

Received 19 October 2015

Received in revised form 13 January 2016

Accepted 7 February 2016

Available online 9 April 2016

\section{Keywords:}

Crack

Inertia effect

Hyperbolic heat conduction

Fourier transform

Singular integral equations

\begin{abstract}
A B S T R A C T
In this paper, the transient temperature fields and the dynamic stress intensity factors of a thermo-elastic strip containing an inner crack parallel to the heated surface under thermal shock are studied. The Biot number of the crack gap, hyperbolic heat conduction theory and equation of motion are considered to investigate the behavior of the temperature fields around the crack and the stress intensity factors. Fourier transform and Laplace transform are used to reduce this mixed boundary value problem. Numerical methods are used to solved the singular integrate equations. Finally, the numerical results are presented illustrating the influence of Biot number, non-Fourier effect and inertia effect on temperature field and stress intensity factors. It is found that the Biot number strongly affect the uniformity of the temperature field and the magnitude of the stress intensity factors. The stress intensity factors have higher amplitude and an oscillating feature comparing to those obtained under conventional Fourier thermal conduction condition and quasi-static hypothesis, which can help to better understand the crack behaviors of advanced materials under thermal impact loading.
\end{abstract}

(c) 2016 Elsevier Ltd. All rights reserved.

\section{Introduction}

Engineering thermo-mechanical structure components are widely used in server thermal loading such as extremely powerful laser impact or thermal barrier coatings (TBCs) used in gas-turbine engines [1,2]. When materials function in the presence of thermal gradients and high heat flux, they are susceptible to submit delamination and fracture. Many articles have studied the failure of such materials after suffering thermal loading [1-5]. In general, authors pay more attention on the cracks parallel to the heating surface (we call it CPHS in short), which play a key role in the spallation failure of materials. Most existing studies about CPHS are based on thermal expansion mismatch between the two different types of the materials or microgeometry defects of materials $[2,3,6]$, both of which rely on the presence of interface. However, CPHS are found not only near the interface of coating materials but also in the coatings far from the interface and non-layered materials [1,7]. There is no doubt that we need an in-depth study to find the source and magnitude of the force which leading to the CPHS.

Classical thermos-elastic stress analysis in a homogeneous body cannot provide tensile stress leading to fractures parallel to the heating surfaces. Therefore, additional driving forces should be considered to explain this class of failures. These driving forces may include the non-Fourier thermal conduction effect [8-10] or the inertia force effect [11-13]. Moreover, the

\footnotetext{
* Corresponding author.
} 


\section{Nomenclature}

a thermal diffusivity

$\mathbf{A}_{i, j} \quad$ function defined in Eq. (A2)

$\mathrm{Bi} \quad$ Biot number

$B_{j} \quad$ function defined in Eq. (39)

$C_{p} \quad$ specific heat capacity

$C_{i} \quad$ unknown coefficients

$D_{i} \quad$ unknown coefficients

e mathematical constant

E Young's modulus

$f_{1}, f_{2} \quad$ dislocation function

$h$ heat transfer coefficient

i imaginary unit

$i, j \quad$ subscript

$k$ thermal conductivity

$k() \quad$ function defined in Eq. (24)

$K_{i, j} \quad$ function defined in Eq. (A4)

$l_{a}, l_{b} \quad$ distance between the crack and the boundary

$L a, L b$ dimensionless distance between the crack and the boundary

$m \quad$ variable defined in Eq. (18)

$m_{0} \quad$ variable defined in Eq. (22)

$p \quad$ Laplace variable

$\vec{q} \quad$ heat flux vector

$r \quad$ crack length

$t$ time

$T$ temperature

$T_{0} \quad$ initial temperature

$T_{\infty} \quad$ temperature of the surrounding external environment of the strip

$u, v \quad$ displacement

$\bar{u}, \bar{v} \quad$ displacement in Laplace space

$\tilde{\bar{u}}, \tilde{\bar{v}} \quad$ displacement in Laplace-Fourier space

$V \quad$ elastic wave velocity

$W_{i} \quad$ function defined in Eq. (A4)

$x, y \quad$ coordinate

$X, Y \quad$ dimensionless coordinate

$\nabla \quad$ Laplace operator

Greek symbols

$\alpha \quad$ coefficient of linear thermal expansion

$\beta_{i} \quad$ function defined in Eq. (34)

$\gamma_{j} \quad$ function defined in Eq. (A2)

$\delta() \quad$ Dirac delta function

$\varepsilon \quad$ strain

$\eta \quad$ integral variable

$\theta \quad$ dimensionless temperature

$\lambda_{i} \quad$ function defined in Eq. (37)

$v \quad$ Poisson's ratio

$\xi \quad$ Fourier variable

$\pi \quad$ circumference ratio

$\rho \quad$ density

$\sigma_{x}, \sigma_{y} \quad$ normal stress

$\tau \quad$ dimensionless time

$\tau_{0} \quad$ thermal relaxation time

$\tau_{1} \quad$ Fourier factor

$\tau_{2} \quad$ inertia factor

$\tau_{x y} \quad$ shear stress

$\varphi() \quad$ dislocation density function

$\Phi() \quad$ function defined in Eq. 25

$\omega_{i} \quad$ function defined in Eq. (A1) 
initial heterogeneities such as micro-cracks or inclusions can also develop into macro-cracks under thermal shock [2,3]. In fact, these micro-cracks may act as heat conduction resistance and create temperature gradient around the heterogeneities, favorable to the crack initiation and growth in the material [14-16]. However, the interaction of all these factors leads to a very complex initial-boundary value problem and has not been thoroughly investigated in the literature so far [1].

The main objective of the present work is to perform a theoretical-numerical analysis that allows the assessment of all these driving forces in the thermal stress induced fracture process, especially in the propagation of a CPHS. To this end, we focus our attention on stress analysis in a cracked bi-dimensional strip under a thermal load. The Laplace and Fourier transforms were used as principal theoretical tool in this analysis. First of all, for the boundary conditions, heat transfer coefficient is introduced to the crack surfaces in order to modulate the heat transfer across the crack. A dimensionless parameter, the Biot number is therefore introduced to describe the influence of the heat insulation of the crack. Secondly, a higher-order time-derivative term specifying the non-Fourier effect is added to the Fourier heat conduction equation. Consequently, a hyperbolic heat conduction equation should be resolved instead of the classical parabolic equations. Finally, for the stress field, equations of motion are considered by introducing the inertia effect instead of the equilibrium equations. The resolution of such an initial-boundary value problem permits us to assess the role of each fracture driving force and their interaction. Then the numerical results on the stress intensity factors of the crack are presented and discussed accordingly, followed by some brief concluding remarks.

\section{Statement of the problem and basic equations}

We consider a thin plate under transient thermal loading which contains a straight crack of length $2 r$ parallel to the free surface, as shown in Fig. 1. A Cartesian coordinate system is established with the $x$-axis coincided with the crack plane and the origin at the crack center. We assume that the material is homogeneous, isotropic and linearly elastic. In this work, only thermal loading is considered in evaluating the temperature and stress fields. The initial temperature is assumed to be uniform and equals to $T_{0}$ in the plate. Then the free surface $y=-l_{a}$ is suddenly heated to a fixed temperature $T_{\infty}$, while the free surface $y=l_{b}$ stays unchanged, where $l_{a}$ and $l_{b}$ are strictly positive real. The heat transfer coefficients of the crack surface are assumed to be $h$. Additionally, the thermal-elastic coupling is neglected in this study.

In order to assess the influence of the non-Fourier and the dynamic effect of the thermal shock, we adopt the hyperbolic heat conduction equations in temperature field evaluation and introduce the inertia effect in stress field to the equilibrium equations.

In the following, we present the detailed mathematical formulation for the resolution of the above-defined thermal shock problem.

\subsection{Heat conductions}

The famous Fourier's law of heat conduction presents a linear relationship between the heat flux $(\vec{q})$ through a material and the gradient of temperature $(T)$, whose differential form is $\vec{q}=-k \nabla T$, where $k$ is the material thermal conductivity and $\nabla$ is the gradient operator. In principle, however, the Fourier's law leads to an unphysical infinite heat propagation speed within a continuum for transient heat conduction processes because of its parabolic characteristics. To overcome this contradiction, a hyperbolic model, named the Cattaneo-Vernotte model, has been proposed by introducing a time-dependent term into the Fourier equation [17,18]. The introduced time-derivative term describes a wave nature of heat propagation at a finite speed, which has been proved in both theory and experiments $[10,18,19]$. The governing equation of nonFourier thermal conduction writes

$$
a \cdot \nabla^{2} T=\frac{\partial T}{\partial t}+\tau_{0} \frac{\partial^{2} T}{\partial t^{2}}
$$

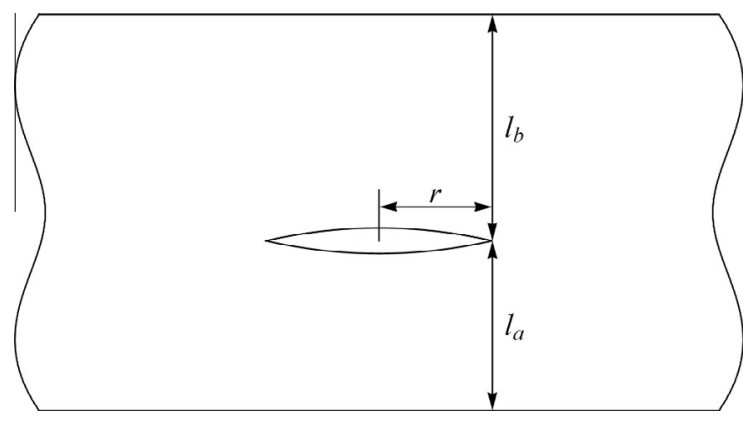

Fig. 1. Model and coordinates. 
where $a=\frac{k}{\rho c_{p}}$ stands for thermal diffusivity, $t$ is he time, $\rho$ and $C_{p}$ are the mass density and the specific heat capacity, respectively, $\nabla$ is the Laplace differential operator and $\tau_{0}$ is the thermal relaxation time.

In $2 \mathrm{D}$ problems, we introduce the following dimensionless coordinates, time and temperature

$$
(X, Y)=\frac{(x, y)}{r}, \quad \tau=\frac{a t}{r^{2}}, \quad \theta=\frac{T-T_{0}}{T_{\infty}-T_{0}}
$$

where $T_{0}$ is the initial temperature of the plate and the governing Eq. (1) takes the following dimensionless forms

$$
\nabla^{2} \theta=\frac{\partial \theta}{\partial \tau}+\tau_{1} \frac{\partial^{2} \theta}{\partial \tau^{2}}
$$

where $\tau_{1}$ is the normalized thermal relaxation time defined as:

$$
\tau_{1}=\frac{a \tau_{0}}{r^{2}}
$$

Here the gradient operator $\nabla$ is related to the dimensionless coordinates $X$ and $Y$.

Referring to Fig. 1, the hyperbolic heat Eq. (3) is subjected to the following boundary and initial conditions written in dimensionless forms

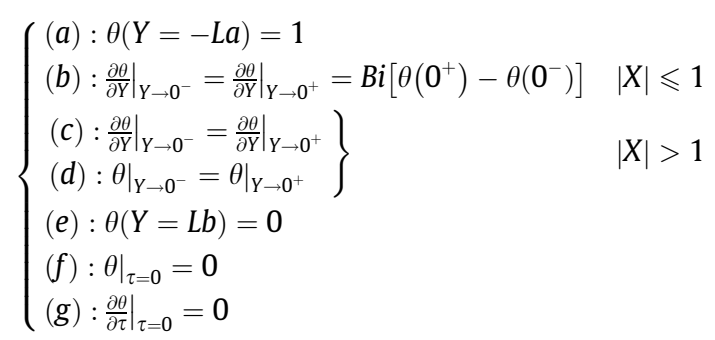

where $B i$ is the Biot number, which gives a simple index of the ratio of the heat transfer resistances inside of and at the crack surface, is defined as:

$$
B i=\frac{h r}{k}=\frac{1 / k}{1 / h r}
$$

with $h$ being the heat transfer coefficient across the crack, which is the proportionality coefficient between the heat flux and the thermodynamic driving force for the flow of heat. The numerator and the denominator of the right part of Eq. (6) stand for the thermal resistance of the internal material and the thermal resistance of the crack surfaces, respectively.

\subsection{Thermal-elastic field equations}

In the following, we focus on attention in resolving the plane-stress thermal-elastic problem under the plane stress hypothesis

$$
\sigma_{z z}=\tau_{x z}=\tau_{y z}=0
$$

The basic equations of plane thermal stress problems for nonhomogeneous isotropic elastic body are:

(1) Equations of motion including the inertia effect:

$$
\sigma_{i j, j}=\rho \ddot{u}
$$

(2) Strain-displacement relations:

$$
\varepsilon_{i j}=\frac{1}{2}\left(u_{i, j}+u_{j, i}\right)
$$

(3) Thermal-elastic stress-strain relations:

$$
\left\{\begin{array}{l}
\sigma_{x x}=\frac{E}{1-v^{2}}\left[\varepsilon_{x x}+v \varepsilon_{y y}-(1+v) \alpha\left(T-T_{0}\right)\right] \\
\sigma_{y y}=\frac{E}{1-v^{2}}\left[v \varepsilon_{x x}+\varepsilon_{y y}-(1+v) \alpha\left(T-T_{0}\right)\right] \\
\sigma_{x y}=\frac{E}{1+v} \varepsilon_{x y}
\end{array}\right.
$$

where $E, v$ and $\alpha$ are the Young modulus, the Poisson ratio and the coefficient of linear thermal expansion, respectively. The index $i, j$ refers to the coordinate $x, y$. The index $i, j$ refers to the coordinate $x, y$. It is to notice that in this paper, the italic letter " $i$ " is used as a subscript. It should not be confounded with the regular script " $i$ ", which stands for the imaginary unit. 
Besides Eq. (2), we introduce some other dimensionless quantities:

$$
\sigma_{I J}=\frac{(1-v) \sigma_{i j}}{E \alpha\left(T_{\infty}-T_{0}\right)}, \quad(u, v)=\frac{\left(u_{x}, u_{y}\right)}{r(1+v) \alpha\left(T_{\infty}-T_{0}\right)}
$$

where the index $I, J$ refer to the dimensionless coordinate $X, Y$.

By introducing the dimensionless variables defined in (11) into (9) and (10), we obtain stress-strain relationships in dimensionless form:

$$
\left\{\begin{array}{l}
\sigma_{X X}=\frac{\partial u}{\partial X}+v \frac{\partial v}{\partial Y}-\theta \\
\sigma_{Y Y}=v \frac{\partial u}{\partial X}+\frac{\partial v}{\partial Y}-\theta \\
\tau_{X Y}=\frac{1-v}{2}\left(\frac{\partial u}{\partial Y}+\frac{\partial v}{\partial X}\right)
\end{array}\right.
$$

The equations of motion become:

$$
\left\{\begin{array}{l}
\frac{\partial \sigma_{X X}}{\partial X}+\frac{\partial \tau_{X Y}}{\partial Y}=\tau_{2}^{2} \frac{(1-v)^{2}}{1-2 v} \frac{\partial^{2} u}{\partial \tau^{2}} \\
\frac{\partial \tau_{X Y}}{\partial X}+\frac{\partial \sigma_{Y Y}}{\partial Y}=\tau_{2}^{2} \frac{(1-v)^{2}}{1-2 v} \frac{\partial^{2} v}{\partial \tau^{2}}
\end{array}\right.
$$

By substituting the equations of motion (13) with Eq. (12), and by using the dimensionless quantities, we obtain:

$$
\left\{\begin{array}{l}
\frac{\partial^{2} u}{\partial X^{2}}+\frac{1+v}{2} \frac{\partial^{2} v}{\partial X \partial Y}+\frac{1-v}{2} \frac{\partial^{2} u}{\partial Y^{2}}-\tau_{2}^{2} \frac{(1-v)^{2}}{1-2 v} \frac{\partial^{2} u}{\partial \tau^{2}}=\frac{\partial \theta}{\partial X} \\
\frac{\partial^{2} v}{\partial Y^{2}}+\frac{1+v}{2} \frac{\partial^{2} u}{\partial X \partial Y}+\frac{1-v}{2} \frac{\partial^{2} v}{\partial X^{2}}-\tau_{2}^{2} \frac{(1-v)^{2}}{1-2 v} \frac{\partial^{2} v}{\partial \tau^{2}}=\frac{\partial \theta}{\partial Y}
\end{array}\right.
$$

where $\tau_{2}=\frac{a}{V r}$ with $V=\sqrt{\frac{E(1-v)}{\rho(1+v)(1-2 v)}}$ being the stress wave velocity.

The dimensionless parameter $\tau_{2}$, which we named "inertia factor", plays an important role in describing the dynamic behavior of a cracked solid under thermal loading. The physical implication of this parameter in stress concentration near the crack tips will be discussed afterward in this paper.

In the present problem, the mechanical boundary conditions can be expressed as follows:

$$
\left\{\begin{array}{ll}
(a): \tau_{X Y}(X,-L a)=\sigma_{Y Y}(X,-L a)=0 & \\
(b): \tau_{X Y}(X, L b)=\sigma_{Y Y}(X, L b)=0 & \\
(c): \tau_{X Y}(X, 0)=\sigma_{Y Y}(X, 0)=0 & |X|<1 \\
(d): \tau_{X Y}\left(X, 0^{+}\right)=\tau_{X Y}\left(X, 0^{-}\right) \\
(e): \sigma_{Y Y}\left(X, 0^{+}\right)=\sigma_{Y Y}\left(X, 0^{-}\right) \\
(f): u\left(X, 0^{-}\right)=u\left(X, 0^{+}\right) \\
(g): v\left(X, 0^{-}\right)=v\left(X, 0^{+}\right)
\end{array}\right\} \quad|X| \geqslant 1
$$

\section{Temperature field}

In the following, we present the resolution procedure of the above-defined thermal-elastic equations. The analytical-numerical methods used in [20,21] are essentially followed in this work. Globally speaking, the Laplace and the Fourier transform techniques are used in resolving the problem [22]. Science the inertia effect is introduced in the equilibrium equations, the resolution procedure becomes more complex. Special resolution techniques are developed in this paper.

The temperature field in the cracked plate can be obtained by solving the governing Eq. (3) under initial and boundary conditions (5). Applying the Laplace transform to (3) and (5) leads to:

$$
\nabla^{2} \bar{\theta}=\left(p+\tau_{1} p^{2}\right) \bar{\theta}
$$

and

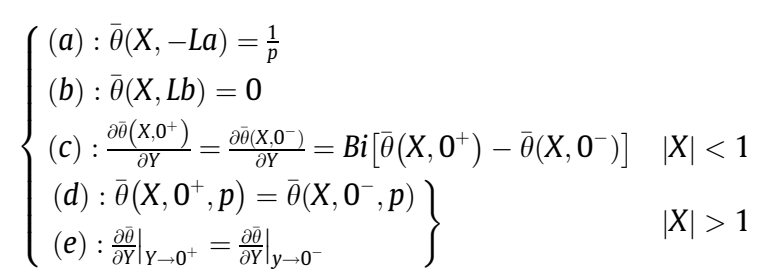

where $\bar{\theta}(X, Y, p)=\int_{0}^{\infty} \theta(X, Y, \tau) e^{-p \tau} d \tau$ is the Laplace transform of dimensionless temperature.

The general solution of (16) can be solved by using Fourier transform method. Temperature in Laplace-Fourier space can be written as: 


$$
\tilde{\bar{\theta}}(\xi, Y, p)= \begin{cases}D_{1}(\xi) e^{-m Y}+D_{2}(\xi) e^{m Y} & -L a<Y<0 \\ D_{3}(\xi) e^{-m Y}+D_{4}(\xi) e^{m Y} & 0<Y<L b\end{cases}
$$

where $m=\sqrt{\xi^{2}+p+\tau_{1} p^{2}}$ and $\tilde{\bar{\theta}}(\xi, Y, p)=\int_{-\infty}^{\infty} \bar{\theta}(X, Y, p) e^{i \xi x} d x, D_{i}(\xi)(i=1, \ldots, 4)$ are unknown parameters to be determined.

Now we introduce the following density function in Laplace space:

$$
\bar{\phi}(X)=\left.\frac{\partial \bar{\theta}}{\partial X}\right|_{Y \rightarrow 0^{+}}-\left.\frac{\partial \bar{\theta}}{\partial X}\right|_{Y \rightarrow 0^{-}}
$$

It is clear that from the boundary condition (c) in (17), we have:

$$
\int_{-1}^{1} \bar{\phi}(X) d X=0
$$

and

$$
\bar{\phi}(X)=0,|X|>1
$$

By applying the boundary conditions (17) and the density function (19), the temperature field in Laplace space can be expressed as:

$$
\bar{\theta}=\left\{\begin{array}{l}
\frac{1}{2 \pi} \int_{0}^{\infty} \frac{\mathrm{ie}^{-m y}\left(1+\mathrm{e}^{2 L b m}\right)\left(1-\mathrm{e}^{2 m(L a+Y)}\right)}{\left(-1+\mathrm{e}^{2(L a+L b) m}\right) \xi} \tilde{\bar{\phi}} \cos (\xi X) d \xi+\frac{\mathrm{e}^{m_{0}(L a+2 L b-Y)}-\mathrm{e}^{m_{0}(L a+Y)}}{\left(-1+\mathrm{e}^{2(L a+L b) m_{0}}\right) p}-L a<Y \leqslant 0 \\
\frac{1}{2 \pi} \int_{0}^{\infty} \frac{\mathrm{ie}^{-m y}\left(1+\mathrm{e}^{2 L a m}\right)\left(\mathrm{e}^{2 L b m}-\mathrm{e}^{2 m Y}\right)}{\left(-1+\mathrm{e}^{2(L a+L b) m}\right) \xi} \tilde{\bar{\phi}} \cos (\xi X) d \xi+\frac{\mathrm{e}^{m_{0}(L a+2 L b-Y)}-\mathrm{e}^{m_{0}(L a+Y)}}{\left(-1+\mathrm{e}^{2(L a+L b) m_{0}}\right) p} 0<Y<L b
\end{array}\right.
$$

where $\tilde{\bar{\phi}}=\int_{-\infty}^{\infty}\left(\int_{0}^{\infty} \phi e^{-p \tau} d \tau\right) e^{i \xi X} d \xi$ is the Fourier transform of Laplace transform of $\phi ; m=\sqrt{\xi^{2}+p+\tau_{1} p^{2}}$ and $m_{0}=\sqrt{p+\tau_{1} p^{2}}$.

Substituting (22) into (c) in (17), we get the singular integral equation for $\phi(X)$ as follows:

$$
\int_{-1}^{1} \bar{\phi}(\eta)\left[\frac{1}{\eta-X}+k(X, \eta)\right] d \eta=\frac{2 \pi m_{0} \mathrm{e}^{L a m_{0}}\left(1+\mathrm{e}^{2 L b m_{0}}\right)}{\left(-1+\mathrm{e}^{2 m_{0}(L a+L b)}\right) p}
$$

where

$$
k(X, \eta)=\int_{0}^{\infty}\left[1-\frac{2 B i}{\xi}-\frac{\left(1+\mathrm{e}^{2 L a m}\right)\left(1+\mathrm{e}^{2 L b m}\right) m}{\left(-1+\mathrm{e}^{2(L a+L b) m}\right) \xi}\right] \sin \xi(X-\eta) d \xi
$$

The integral Eq. (23) under the singles-value condition (20) has the following form of solution [23]

$$
\bar{\phi}(X)=\frac{\bar{\Phi}(X)}{\sqrt{1-X^{2}}}
$$

where $\bar{\Phi}(X)$ is bounded and continuous on the interval [-1, 1]. Following the numerical techniques proposed by [23], Eqs (20) and (23) can be solved at discrete points from the algebraic equations system:

$$
\left\{\begin{array}{l}
\sum_{j=1}^{n} \frac{\pi}{n} \bar{\Phi}\left(\eta_{j}\right)\left[\frac{1}{\eta_{j}-X_{i}}+k\left(X_{i}, \eta_{j}\right)\right]=\frac{2 \pi m_{0} \mathrm{e}^{L a m_{0}}\left(1+\mathrm{e}^{2 L b m} 0\right)}{\left(-1+\mathrm{e}^{2 m_{0}(L a+L b)}\right) p} \\
\sum_{j=1}^{n} \frac{\pi}{n} \bar{\Phi}\left(\eta_{j}\right)=0
\end{array}\right.
$$

where the Gauss-Chebyshev points are defined as

$$
\eta_{j}=\cos \left(\frac{2 j-1}{2 n} \pi\right) \quad j=1,2, \ldots, n
$$

and

$$
X_{i}=\cos \left(\frac{i \pi}{n}\right) \quad i=1,2, \ldots, n-1
$$

Once the function $\bar{\Phi}(X)$ is obtained, the temperature fields in $p$-plane can be calculated by using Eqs. (22) and applying the Chebyshev quadrature for integration.

Temperature in time domain can be given by applying the inverse Laplace transform, as detailed in [20]. 


\section{Thermal stress}

Once the temperature field in Laplace space is found, it is introduced to the equations of motion (14) to determine the displacement and stress fields. To this end, we first write the equations of motion (14) and the boundary conditions (15) in Laplace space by means of the Laplace transform. Then the Fourier transform with respect to $X$ coordinate is applied to these equations such that a non-linear ordinary differential equation system in $Y$-coordinate can be obtained in FourierLaplace space. These differential equations are resolved by using appropriate numerical methods. Inverse Fourier transform then inverse Laplace transform of these solutions provide the displacement and stress fields with which some important measures such as the dynamic stress intensity factors at the crack tips, the crack opening distance etc. can be obtained.

\subsection{Integral transformation}

Firstly, using the Laplace transform to Eqs. (14) and (15), we obtain:

$$
\begin{aligned}
& \left\{\begin{array}{l}
\frac{\partial^{2} \bar{u}}{\partial X^{2}}+\frac{1+v}{2} \frac{\partial^{2} \bar{v}}{\partial X \partial Y}+\frac{1-v}{2} \frac{\partial^{2} \bar{u}}{\partial Y^{2}}-\tau_{2}^{2} \frac{(1-v)^{2}}{1-2 v} p^{2} \bar{u}=\frac{\partial \bar{\theta}}{\partial X} \\
\frac{\partial^{2} \bar{v}}{\partial Y^{2}}+\frac{1+v}{2} \frac{\partial^{2} \bar{u}}{\partial X \partial Y}+\frac{1-v}{2} \frac{\partial^{2} \bar{v}}{\partial X^{2}}-\tau_{2}^{2} \frac{(1-v)^{2}}{1-2 v} p^{2} \bar{v}=\frac{\partial \bar{\theta}}{\partial Y}
\end{array}\right. \\
& \left\{\begin{array}{l}
(a): \overline{\tau_{X Y}}(X,-L a)=\overline{\sigma_{Y Y}}(X,-l)=0 \\
(b): \overline{\tau_{X Y}}(X, L b)=\overline{\sigma_{Y Y}}(X, L b)=0 \\
(c): \bar{\tau}_{X Y}(X, 0)=\overline{\sigma_{Y Y}}(X, 0)=0 \\
(d): \overline{\tau_{X Y}}\left(X, 0^{+}\right)=\overline{\tau_{X Y}}\left(X, 0^{-}\right) \\
(e): \overline{\sigma_{Y Y}}\left(X, 0^{+}\right)=\overline{\sigma_{Y Y}}\left(X, 0^{-}\right) \\
(f): \bar{u}\left(X, 0^{+}\right)=\bar{u}\left(X, 0^{-}\right) \\
(g): \bar{v}\left(X, 0^{+}\right)=\bar{v}\left(X, 0^{-}\right)
\end{array}\right\} \quad|X| \geqslant 1
\end{aligned}
$$

where $(\bar{u}, \bar{v})=\int_{0}^{\infty}(u, v) e^{-p \tau} d \tau$ and $\bar{\sigma}_{I J}=\int_{0}^{\infty} \sigma_{I J} e^{-p \tau} d \tau$ are the Laplace transform of the displacement components and stress components, respectively.

Then applying the Fourier transform to Eqs. (29) and (30) gives

$$
\begin{aligned}
& \left\{\begin{array}{l}
\frac{d^{2} \tilde{\tilde{u}}}{d Y^{2}}+\beta_{1} \frac{d \tilde{\bar{v}}}{d Y}+\beta_{2} \tilde{\bar{u}}=\beta_{3} \tilde{\bar{\theta}} \\
\frac{d^{2} \tilde{\bar{v}}}{d Y^{2}}+\beta_{4} \frac{d \tilde{\bar{u}}}{d Y}+\beta_{5} \tilde{\bar{v}}=\frac{d \tilde{\bar{\theta}}}{d Y}
\end{array}\right. \\
& \left\{\begin{array}{ll}
(a): \widetilde{\overline{\tau_{X Y}}}(-L a)=\widetilde{\overline{\sigma_{Y Y}}}(-L a)=0 & \\
(b): \widetilde{\widetilde{\tau_{X Y}}}(L b)=\widetilde{\overline{\sigma_{Y Y}}}(L b)=0 & \\
(c): \overline{\tau_{X Y}}(0)=\overline{\sigma_{X Y}}(0)=0 & |X| \leqslant 1 \\
(d): \widetilde{\overline{\tau_{X Y}}}\left(0^{+}\right)=\widetilde{\overline{\tau_{X Y}}}\left(0^{-}\right) \\
(e): \widetilde{\overline{\sigma_{Y Y}}}\left(0^{+}\right)=\widetilde{\overline{\sigma_{Y Y}}}\left(0^{-}\right) \\
(f): \tilde{\bar{u}}\left(0^{+}\right)=\tilde{\bar{u}}\left(0^{-}\right) \\
(g): \tilde{\bar{v}}\left(0^{+}\right)=\tilde{\bar{v}}\left(0^{-}\right)
\end{array}\right\} \quad|X|>1 \\
& \left\{\begin{array}{l}
\widetilde{\sigma_{X}}=-i \xi \tilde{\bar{u}}+v \frac{d \tilde{v}}{d Y}-\tilde{\bar{\theta}} \\
\widetilde{\sigma_{Y}}=-i \xi v \tilde{\bar{u}}+\frac{d \tilde{\bar{v}}}{d Y}-\tilde{\bar{\theta}} \\
\widetilde{\overline{\tau_{X Y}}}=\frac{1-v}{2}\left(\frac{d \tilde{\bar{u}}}{d Y}-i \xi \tilde{\bar{v}}\right)
\end{array}\right.
\end{aligned}
$$

where $\tilde{\bar{u}}$ and $\tilde{\bar{v}}$ are the Fourier transform of $\overline{u_{x}}$ and $\overline{u_{y}}$ respectively and $\beta_{i}(i=1,2,3,4,5)$ are given by:

$$
\left\{\begin{array}{l}
\beta_{1}=-\frac{1+v}{1-v} i \xi \\
\beta_{2}=-\frac{2}{1-v}\left(\xi^{2}+\tau_{2}^{2} \frac{(1-v)^{2}}{1-2 v} p^{2}\right) \\
\beta_{3}=-\frac{2 i \xi}{1-v} \\
\beta_{4}=-\frac{1+v}{2} i \xi \\
\beta_{5}=-\left(\frac{1-v}{2} \xi^{2}+\tau_{2}^{2} \frac{(1-v)^{2}}{1-2 v} p^{2}\right)
\end{array}\right.
$$




\subsection{Displacement field and stress field}

By considering the boundary conditions (c), (d) in (32) and the temperature fields, the solution of Eqs. (31), i.e., the displacement field in Laplace-Fourier space can be expressed as:

$$
\left\{\begin{array}{l}
\left\{\begin{array}{l}
\tilde{\tilde{u}}(\xi, Y)=\sum_{i=1}^{4} C_{i}(\xi) e^{\lambda_{i} Y}+\omega_{1} e^{-m Y}+\omega_{2} e^{m Y} \\
\tilde{\bar{v}}(\xi, Y)=\sum_{i=1}^{4} B_{i} C_{i}(\xi) e^{\lambda_{i} Y}+\omega_{3} e^{-m Y}+\omega_{4} e^{m Y}-L a<Y<0
\end{array}\right. \\
\left\{\begin{array}{l}
\tilde{\tilde{u}}(\xi, Y)=\sum_{i=5}^{6} C_{i}(\xi) e^{\lambda_{i} Y}+\omega_{5} e^{-m Y}+\omega_{6} e^{m Y} \quad 0<Y<L b \\
\tilde{\bar{v}}(\xi, Y)=\sum_{i=5}^{6} B_{i} C_{i}(\xi) e^{\lambda_{i} Y}+\omega_{7} e^{-m Y}+\omega_{8} e^{m Y}
\end{array}\right.
\end{array}\right.
$$

where $C_{i}(\xi)(i=1, \ldots, 6)$ are unknown functions to be determined. The expressions of the coefficients $\omega_{i}(i=1, \ldots, 8)$ are given in Eq. (A1). Substituting Eq. (35) into Eq. (31) results in the following equations:

$$
\left\{\begin{array}{l}
\beta_{1} \lambda_{j} B_{j}+\lambda_{j}^{2}+\beta_{2}=0 \\
\left(\lambda_{j}^{2}+\beta_{5}\right) B_{j}+\beta_{4} \lambda_{j}=0
\end{array}\right.
$$

so $\lambda_{i}(i=1, \ldots, 4)$ can be given by the roots of the following equation:

$$
\lambda^{4}+\left(\beta_{2}+\beta_{5}-\beta_{1} \beta_{4}\right) \lambda^{2}+\beta_{2} \beta_{5}=0
$$

and the coefficients $\lambda_{j}(j=5,6,7,8)$ are given by:

$$
\lambda_{j}=\lambda_{j-4}(j=5,6,7,8)
$$

Straightforwardly, the coefficients $B_{j}(j=1,2, \ldots, 8)$ are given by:

$$
B_{j}=-\frac{\lambda_{j}^{2}+\beta_{2}}{\beta_{1} \lambda_{j}}
$$

By substituting the displacement field (35) and temperature field (18) into (33), the stress can be obtained as following:

$$
\left\{\begin{aligned}
\widetilde{\sigma_{X}}= & \sum_{i=i_{0}}^{i_{0}+3}\left(B_{i} \lambda_{i} v-\mathrm{i} \xi\right) C_{i} \mathrm{e}^{\lambda_{i} y}-\mathrm{i} \xi\left(\omega_{1} \mathrm{e}^{-m y}+\omega_{2} \mathrm{e}^{m y}\right)+v\left(-m \omega_{3} \mathrm{e}^{-m y}+m \omega_{4} \mathrm{e}^{m y}\right) \\
& -\left(D_{i_{1}} \mathrm{e}^{-m y}+D_{i_{1}+1} \mathrm{e}^{m y}\right) \\
\widetilde{\sigma_{Y}}= & \sum_{i=i_{0}}^{i_{0}+3}\left(B_{i} \lambda_{i}-\mathrm{i} \xi v\right) C_{i} \mathrm{e}^{\lambda_{i} y}-\mathrm{i} v \xi\left(\omega_{1} \mathrm{e}^{-m y}+\omega_{2} \mathrm{e}^{m y}\right)-m \omega_{3} \mathrm{e}^{-m y}+m \omega_{4} \mathrm{e}^{m y} \\
& -\left(D_{i_{1}} \mathrm{e}^{-m y}+D_{i_{1}+1} \mathrm{e}^{m y}\right) \\
\widetilde{\tau_{X Y}} & =\frac{1-v}{2}\left[\sum_{i=i_{0}}^{i_{0}+3}\left(\lambda_{i}-\mathrm{i} \xi B_{i}\right) C_{i} \mathrm{e}^{\lambda_{i} y}-m \omega_{1} \mathrm{e}^{-m y}+m \omega_{2} \mathrm{e}^{m y}-\mathrm{i} \xi\left(\omega_{3} \mathrm{e}^{-m y}+\omega_{4} \mathrm{e}^{m y}\right)\right]
\end{aligned}\right.
$$

where $i_{0}=1, i_{1}=1$ for $-L a<Y<0$ and $i_{0}=5, i_{1}=3$ for $0<Y<L b$.

\subsection{Integral equations}

We now introduce two dislocation functions $\overline{f_{i}}(X)(i=1,2)$ along the crack line which can be written as follows:

$$
\left\{\begin{array}{l}
\overline{f_{1}}(X)=\frac{\partial\langle\bar{u}\rangle}{\partial X} \\
\overline{f_{2}}(X)=\frac{\partial\langle\bar{v}\rangle}{\partial X}
\end{array}\right.
$$

where $\langle\bar{u}\rangle$ and $\langle\bar{v}\rangle$ denote the jumps of displacements across the crack lips $y=0$ in Laplace space:

$$
\left\{\begin{array}{l}
\langle\bar{u}\rangle=\bar{u}\left(X, 0^{+}\right)-\bar{u}\left(X, 0^{-}\right) \\
\langle\bar{v}\rangle=\bar{v}\left(X, 0^{+}\right)-\bar{v}\left(X, 0^{-}\right)
\end{array}\right.
$$

From the conditions (30), we know that $\overline{f_{i}}(X)(i=1,2)$ also satisfy the single-value conditions: 


$$
\int_{-1}^{1} \overline{f_{i}}(X) d X=0(i=1,2)
$$

and

$$
\overline{f_{i}}(X)=0(i=1,2)|X| \geqslant 1
$$

Physically, Eq. (44) means that the displacements are single-valued for the uncracked portion along $y=0$. By applying the Fourier transforms to Eq. (41), we obtain:

$$
\left\{\begin{array}{l}
\tilde{\widetilde{f_{1}}}(\xi)=-i \xi\left[\tilde{\bar{u}}\left(0^{+}\right)-\tilde{\bar{u}}\left(0^{-}\right)\right] \\
\widetilde{\overline{f_{2}}}(\xi)=-i \xi\left[\tilde{\bar{v}}\left(0^{+}\right)-\tilde{\bar{v}}\left(0^{-}\right)\right]
\end{array}\right.
$$

where by definition and from (43), $\widetilde{f_{1}}$ and $\widetilde{f_{2}}$ are given by:

$$
\widetilde{f_{i}}=\int_{0}^{\infty}\left(\int_{-1}^{1} f_{i} \cdot e^{i \xi X} d X\right) \cdot e^{-p \tau} d \tau \quad i=1,2
$$

By applying the boundary conditions (a)-(e) in (32) and (45), $C_{i}$ can be expressed as

$$
C_{i}=\mathbf{A}_{i, j}^{-1} \gamma_{j}+\frac{1}{i \xi} \mathbf{A}_{i, 7}^{-1} \widetilde{f_{1}}+\frac{1}{i \xi} \mathbf{A}_{i, 8}^{-1} \widetilde{f_{2}} \quad i=1,2, \ldots, 8
$$

where the terms in matrix $\mathbf{A}_{i, j}$ and $\gamma_{j}$ are given in Eqs. (A2) and (A3), respectively.

By substituting (35) into the boundary conditions (c) in (32) and by applying the relationships in (33), it can be shown that $f_{i}(x)(i=1,2)$ satisfy the following equations:

$$
\sum_{j=1}^{2} \int_{-1}^{1} \overline{f_{j}}(\eta) K_{i, j}(X, \eta) d \eta=W_{i} \quad(i=1,2 ; j=1,2)
$$

where $K_{i, j}$ and $W_{i}(i=1,2 ; j=1,2)$ are given in Eq. (A4).

The solution of the integral equations of $f_{i}(x)(i=1,2)$ can be expressed in the following form:

$$
\overline{f_{i}}(X)=\frac{\overline{F_{i}}(X)}{\sqrt{1-X^{2}}} \quad(i=1,2)
$$

Using the Lobatto-Chebyshev method [24-26], we can reduce the integral equations to the following algebraic equations:

$$
\left\{\begin{array}{l}
\sum_{j=1}^{2}\left[\sum_{k=1}^{n} A_{k} K_{1, j}\left(X_{j}, \eta_{k}\right) \overline{F_{j}}\left(\eta_{k}\right)\right]=W_{1}\left(x_{j}\right) \\
\sum_{j=1}^{2}\left[\sum_{k=1}^{n} A_{k} K_{2, j}\left(X_{j}, \eta_{k}\right) \overline{F_{j}}\left(\eta_{k}\right)\right]=W_{2}\left(x_{j}\right) \\
\sum_{k=1}^{n} A_{k} \overline{F_{1}}\left(\eta_{i}\right)=0 \\
\sum_{k=1}^{n} A_{k} \overline{F_{2}}\left(\eta_{i}\right)=0
\end{array}\right.
$$

where

$$
\left\{\begin{array}{l}
\eta_{k}=\cos \frac{(i-1) \pi}{n-1}, k=1,2, \ldots, n ; \\
X_{j}=\cos \frac{(2 j-1) \pi}{2(n-1)}, j=1,2, \ldots, n-1 ; \\
A_{1}=A_{n}=\frac{\pi}{2(n-1)} ; \\
A_{k}=\frac{\pi}{n-1}, k=2,3, \ldots, n-1 .
\end{array}\right.
$$

The functions $F_{1}$ and $F_{2}$ at the crack line can be given by resolving $\overline{F_{1}}$ and $\overline{F_{2}}$ from (50) and performing the inverse Laplace transform. The dynamic stress intensity factors $K_{I}(p)$ and $K_{I I}(p)$ are defined as [27]

$$
\left\{\begin{array}{l}
K_{I}(t)=\mathbf{L}^{-1}\left[-\frac{\left(1-v^{2}\right) \sqrt{\pi}}{8} \overline{F_{2}}(1, p)\right] \\
K_{I I}(t)=\mathbf{L}^{-1}\left[-\frac{(1+v) \sqrt{\pi}}{4} \overline{F_{1}}(1, p)\right]
\end{array}\right.
$$

where the operator $\mathbf{L}^{-1}[$ ] denotes the inverse Laplace transform 


\section{Numerical result and discussions}

The coupled effect of the non-Fourier heat conduction and the inertia forces provides complex stress fields near the crack tips, which depend additionally on the crack position with respect to the boundary of the strip. The influence of both these factors on the principal crack stress concentration characterized mainly by the stress intensity factors (SIFs) and by the crack opening distances (CODs) will be evaluated and discussed in the following sections.

\subsection{Temperature field}

In the analysis of temperature fields and stresses intensity factors, the dimensionless position of the crack in the strip are fixed to be $L_{a}=1$ and $L_{b}=2$. The temperature field can be obtained consecutively. The temperatures in different conditions are depicted in Figs. 2-5.

Fig. 2 shows the influence of the Biot number and the non-Fourier effect on the temperature field. In Fig. 2(a)-(d), thermal relaxation time $\tau_{1}$ is set to be $0,0.2,0.4$ and 0.6 , respectively.

When $B i=0$, as shown in Fig. 2(a), the temperature field corresponding to the case of perfectly insulate crack surfaces, at which condition the temperature differences of the crack surfaces are maximum. When $B i=$ Infinite, as shown in Fig. 2(d), the temperature curves of the upper and lower crack surfaces overlap. In the last case, the temperature field degenerates to that of a strip without crack.

The curves in Fig. 2 show also the influence of the non-Fourier parameter $\tau_{1}$ on the temperature field. The red curves denote $\tau_{1}=0$, which represents the condition of the Fourier thermal conduction. As the increase of $\tau_{1}$, some wave properties appear in the temperature evolution. The peak values of the temperature increase as $\tau_{1}$ increases. Particularly when $\tau_{1}>0$, the maximum normalized temperature could higher than 1 and the minimum normalized temperature could lower than 0 , which is a characteristic phenomenon of the non-Fourier heat conduction. These curves converge to those obtained with the condition of Fourier thermal conduction. The bigger the value of $\tau_{1}$ is, the slower the curve convergence will be.

Fig. 3 shows the Maximum temperature differences between the midpoints of crack surfaces versus $\tau_{1}$ for different Biot number. The increasing of the Biot number would improve the thermal conductivity of the crack gap, which would decrease the temperature differences between the crack surfaces. Whereas the non-Fourier effect increases the peak value of the temperature, especially for the crack surface close to the heating boundary, as shown in Fig. 2, so the maximum temperature difference increasing with $\tau_{1}$.
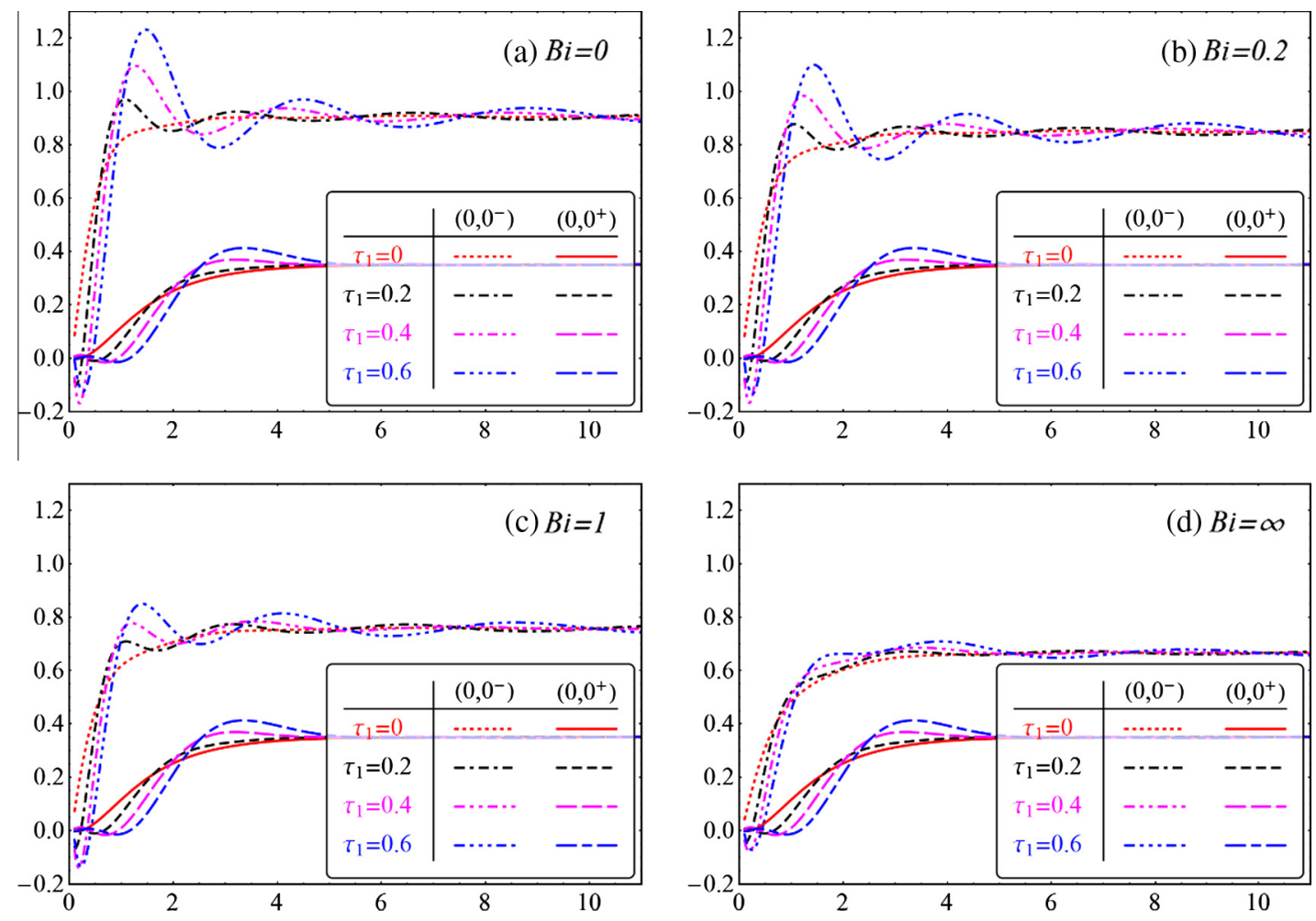

Fig. 2. Temperature evolution at the midpoints of the crack surfaces for different $\tau_{1}$ when (a) $B i=0$, (b) $B i=0.2$, (c) $B i=1$ and (d) $B i=$ Infinity. 


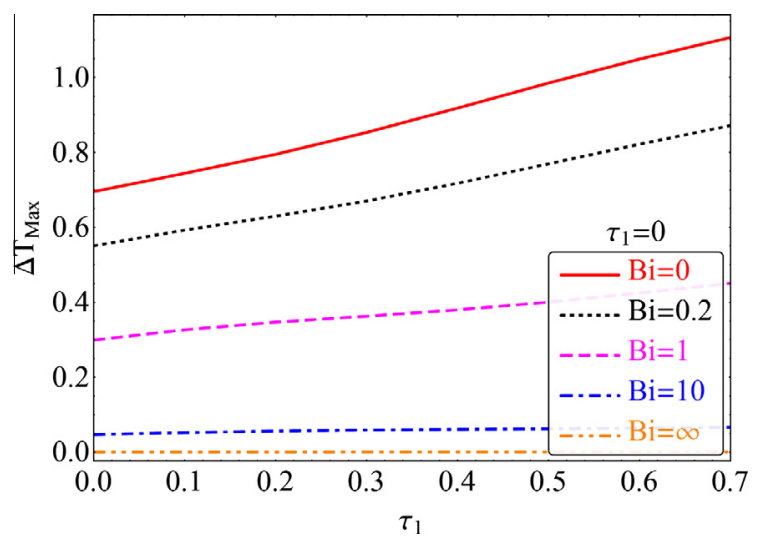

Fig. 3. Temperature differences between the midpoints of the crack surfaces versus $\tau_{1}$ for different Biot number.
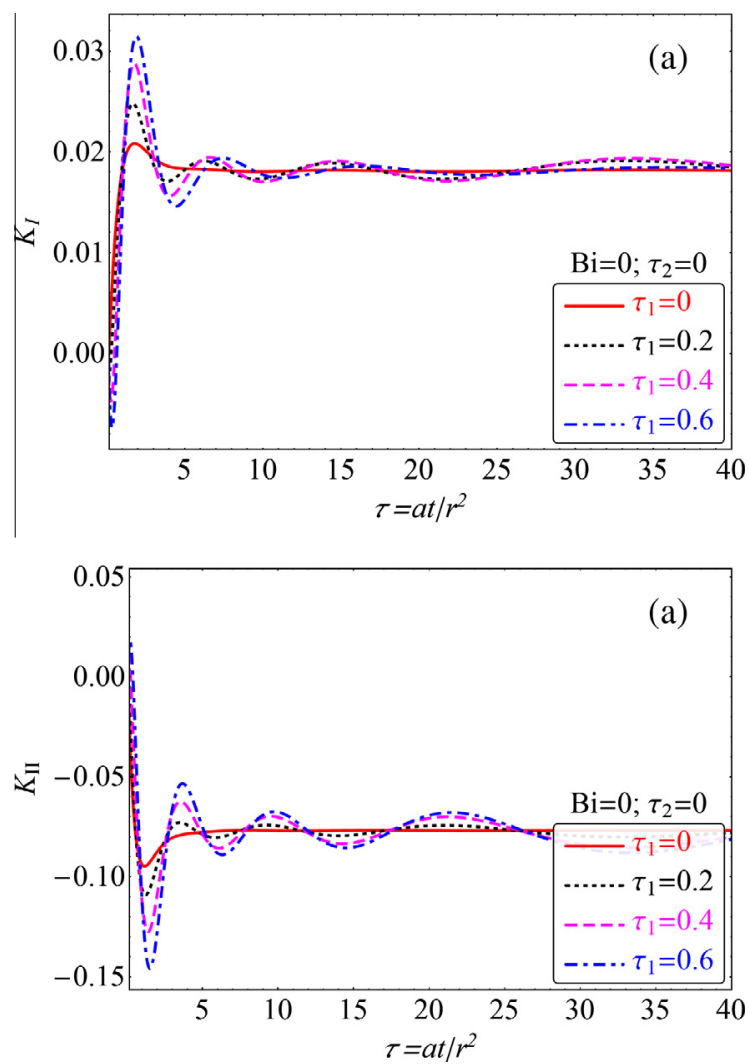

Fig. 4. Normalized stress intensity factors (a) for $K_{I}$ and (b) for $K_{I I}$ for versus dimensionless time for different $\tau_{1}$ when $\tau_{2}=0$.

\subsection{Stress intensity factors (SIFs)}

In this work, the Poisson's ratio is set to be 0.3 for all computations which is close to those of most of the brittle or quasibrittle materials used in engineering applications.

The dynamic stress intensity factors $K_{I}$ and $K_{I I}$ can be evaluated from (52) once we obtain the solutions of the algebraic Eqs. (50) and perform the numerical inverse of Laplace transform. Their normalized values $\left(K_{I}^{*}, K_{I I}^{*}\right)=\frac{\left(K_{I}, K_{I I}\right)(1-v)}{E \alpha\left(T_{\infty}-T_{0}\right) \sqrt{r}}$ are presented in Figs. 4-7. 

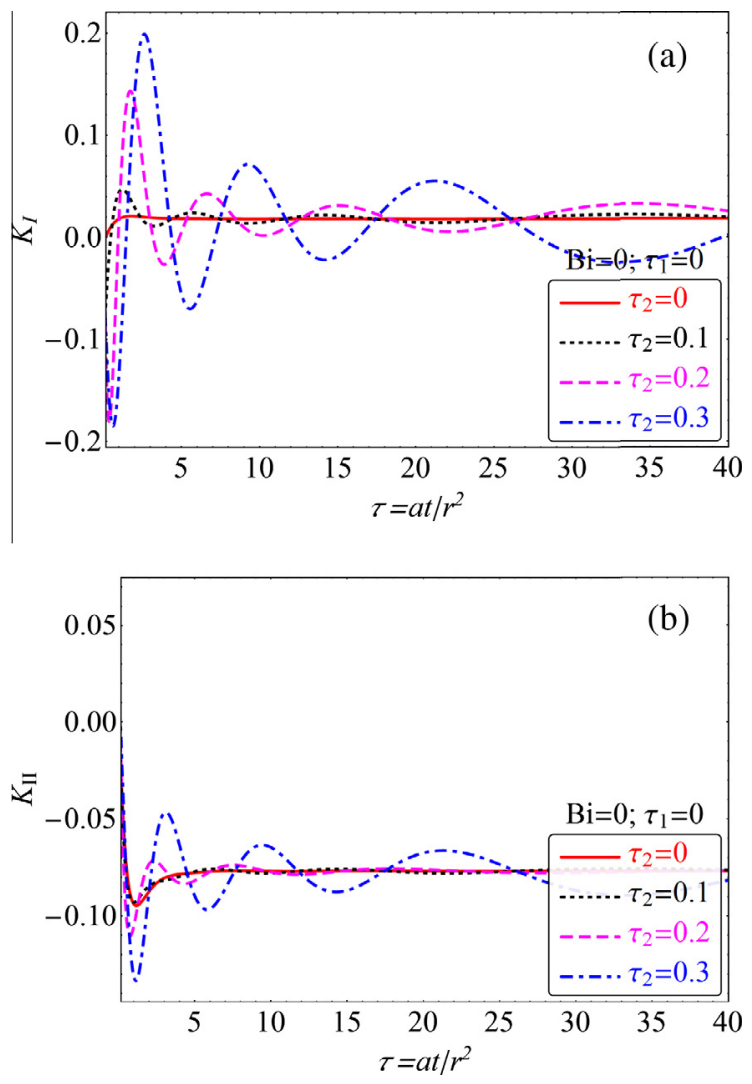

Fig. 5. Normalized stress intensity factors (SIFs) versus dimensionless time for different $\tau_{2}$ when $\tau_{1}=0$.

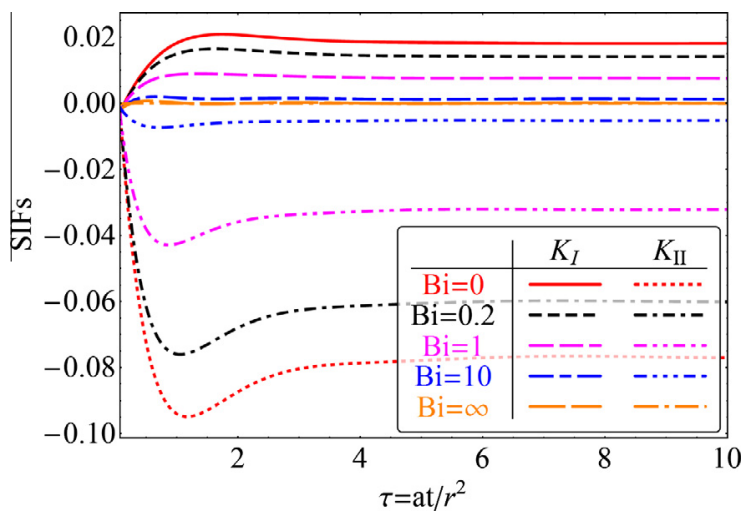

Fig. 6. Variation of SIFs versus dimensionless time for different Biot number when $\tau_{1}=\tau_{2}=0$.

Let us first examine separately the influence of each parameter on the stress intensity factors. Fig. 4 illustrates the evolutions of the SIFs under classical quasi-static condition by taking the non-Fourier thermal conduction effect into account, i.e. $\tau_{2}=0$ and $\tau_{1} \neq 0$.

In this case, the model is identical to the theory developed by Hu and Chen [22] except the thermal shock boundary conditions. In their article both the upper and lower surfaces of the infinite strip suffer from the thermal shock loading with the dimensionless temperature differences to be 2 and 1, respectively. We remark that the trend of the temperature field and SIFs shown in Figs. 2 and 4 exhibit an oscillating feature and agree well with the results reported in the literature [8,22,28].

The influence of the thermal inertia factor $\tau_{2}$ on the normalized dynamic SIFs is depicted in Fig. 5 by neglecting the nonFourier effect, i.e. by fixing $\tau_{1}=0$. From this figure, we can clearly observe the influence of this parameter on the dynamic 


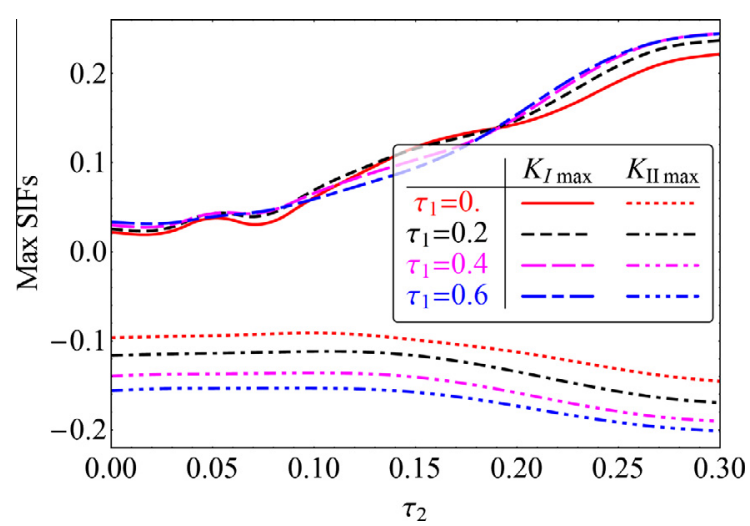

Fig. 7. Variation of maximum SIFs versus $\tau_{2}$ for different $\tau_{1}$ when Biot number is set to be 0 .

SIFs: Firstly, the impact of $\tau_{2}$ is much stronger on $K_{\mathrm{I}}^{*}$ than on $K_{\mathrm{II}}^{*}$ in the range of $\tau_{2} \in[0,0.3]$. Secondly, as expected, the amplitudes of the SIFs increase with $\tau_{2}$. Moreover, similar to the case when $\tau_{1}>0$, the time evolution of the SIFs presents an oscillating features when $\tau_{2}$ is large. It is to notice that the dynamic SIF $K_{\mathrm{I}}^{*}$ can be positive or negative when $\tau_{2}>0$. This anomaly comes from the fact that the crack lip contact under compression is not taken into account in this study.

Fig. 6 shows the effect of the Biot number on the dynamic SIFs in the case of Fourier heat conduction $\left(\tau_{1}=0\right)$ and quasistatic thermal stress $\left(\tau_{2}=0\right)$. It is clear that both the $K_{\mathrm{I}}$ and $K_{\mathrm{II}}$ have the peak value when the crack gap is heat insulating. As the growing of the Biot number, the temperature difference between the crack surfaces decreases, as shown in Fig. 2. Consequently, the absolute values of SIFs gradually decrease and finally approach to zero when the Biot number tends to infinity.

In practice, the peak values of the dynamic SIFs during the time evaluation are most significant in predicting the crack growth. The peak values of $K_{1}^{*}$ evaluated for different $\tau_{1}$ and $\tau_{2}$ are plotted in Fig. 7, in which the Biot number is set to be 0 . Overall, the maximum absolute value increase with $\tau_{1}$ and $\tau_{2}$. The non-Fourier effect has bigger impact to $K_{\mathrm{II}}$ while the inertial effect affects more on $K_{\mathrm{I}}$. In particular, the maximum $K_{\mathrm{I}}$ increases more than 10 times as $\tau_{2}$ grows from 0 to 0.3 .

In most engineering problems the length of engineering cracks is considered to be larger than $1 \mathrm{~mm}$. In this condition the value of $\tau_{2}$ is normally less than 0.01 and the magnitude of $\tau_{2}$ is small enough to be ignored. However, according to the definition of $\tau_{2}$, its value will be greatly increased as the decreasing of the crack length. For example, the $\tau_{2}$ value of gold will reach up to 0.115 when the crack length is $1 \mu \mathrm{m}$. In fact, the micro-cracks smaller than $1 \mu \mathrm{m}$ do exist in some coating materials. $[29,30]$, the inertia effect should be considered and will play an important role in the thermal shock damage process.

\section{Conclusions}

In this paper, the insulation of the crack gap, the non-Fourier heat conduction condition and the inertia effect are considered to investigate the transient thermal stress problem of an insulated crack parallel to the boundary of a thermal-elastic plate under thermal shock loading. The mixed boundary value problem is formulated in terms of Laplace and Fourier transforms techniques. The established singular integral equations are solved by using the Gauss integrate method. Temperature and dynamic stress intensity factors in the time domain are obtained by using numerical inverse Laplace transform. The Temperature field and dynamic stress intensity factors are evaluated for different values of parameters $B i, \tau_{1}$ and $\tau_{2}$.

The magnitude of Biot number of the crack gaps has great influence for both the temperature field and stress intensity factors. The numerical results reveal that the influence of the crack to the temperature and stress field can be neglect when $B i>100$. In other words, the increasing of Biot number would increase the resistance of cracks growth. This result might be useful in designing new materials.

The magnitude of thermal relaxation time and the strength of non-Fourier effect have been investigated by many authors. General speaking, the non-Fourier effect is unneglectable in some non-homogeneous materials [17] and biological materials, such as human tissue [31].

The inertia effect is negligible when the crack length is in millimeter scale or larger. However, when the scale of interest is small to micron scale, the inertia effect will play an important role in the thermal shock damage of materials.

\section{Acknowledgements}

This work was supported by the funding from the Programs in the National Natural Science Foundations of China (Grants 11232013 and 11472285) and the French ANR program T-Shock ANR-10-INTB-0915. 


\section{Appendix A}

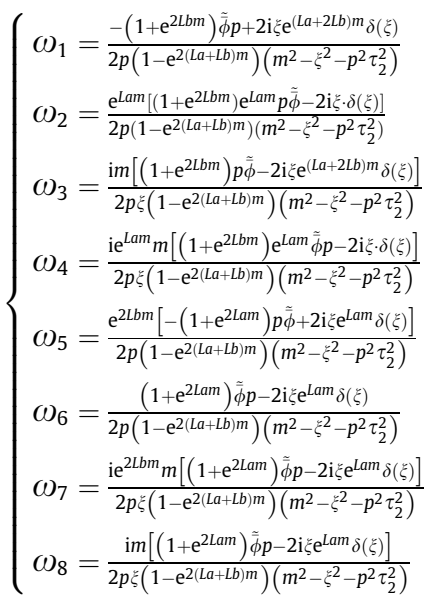

where $\delta(\xi)=\left\{\begin{array}{ll}\infty, & \xi=0 \\ 0, & \xi \neq 0\end{array}\right.$.

$$
\begin{aligned}
& \begin{array}{l}
\mathbf{A}_{1, j}= \begin{cases}\left(\mathrm{i} \xi v+B_{j} \lambda_{j}\right) \mathrm{e}^{-\lambda_{j} L a} & j=1,2,3,4 \\
0 & j=5,6,7,8\end{cases} \\
\mathbf{A}_{2 j}= \begin{cases}\left(\mathrm{i} \xi B_{j}+\lambda_{j}\right) \mathrm{e}^{-\lambda_{j} L a} & j=1,2,3,4 \\
0 & j=5,6,7,8\end{cases}
\end{array} \\
& \mathbf{A}_{3, j}= \begin{cases}\mathrm{i} \xi v+B_{j} \lambda_{j} & j=1,2,3,4 \\
-\mathrm{i} \xi v-B_{j} \lambda_{j} & j=5,6,7,8\end{cases} \\
& \mathbf{A}_{4, j}= \begin{cases}\mathrm{i} \xi B_{j}+\lambda_{j} & j=1,2,3,4 \\
-\mathrm{i} \xi B_{j}-\lambda_{j} & j=5,6,7,8\end{cases} \\
& \mathbf{A}_{5, j}= \begin{cases}0 & j=1,2,3,4 \\
\left(\mathrm{i} \xi \nu+B_{j} \lambda_{j}\right) \mathrm{e}^{\lambda_{j} L b} & j=5,6,7,8\end{cases} \\
& \mathbf{A}_{6, j}= \begin{cases}0 & j=1,2,3,4 \\
\mathrm{i} \xi B_{j}+\lambda_{j} & j=5,6,7,8\end{cases} \\
& \mathbf{A}_{7, j}= \begin{cases}-1 & j=1,2,3,4 \\
1 & j=5,6,7,8\end{cases} \\
& \mathbf{A}_{8, j}= \begin{cases}-B_{i} & j=1,2,3,4 \\
B_{i} & j=5,6,7,8\end{cases} \\
& \gamma_{j}=\left[\begin{array}{l}
\frac{(1-v) \xi^{2}+p^{2} \tau_{2}^{2}}{p\left(-m^{2}+\xi^{2}+p^{2} \tau_{2}^{2}\right)} \delta(\xi) \\
-\frac{2 m\left[p\left(\mathrm{e}^{\mathrm{e} a m}+\mathrm{e}^{(L a+2 L b) m}\right) \tilde{\bar{\phi}}-\mathrm{i} \xi\left(1+\mathrm{e}^{2(L a+L b) m}\right) \delta(\xi)\right]}{\left(-1+\mathrm{e}^{2(L a+L b) m}\right) p\left(-m^{2}+\xi^{2}+p^{2} \tau_{2}^{2}\right)} \\
\frac{\mathrm{i} \tilde{\bar{\phi}}\left((1-v) \xi^{2}+p^{2} \tau_{2}^{2}\right)}{\xi\left(m^{2}-\xi^{2}-p^{2} \tau_{2}^{2}\right)} \\
0 \\
0 \\
-\frac{2 \mathrm{e}^{L b m} m\left[\left(1+\mathrm{e}^{2 L a m}\right) \tilde{\bar{\phi}} p-2 \mathrm{ie}^{L a m} \delta(\xi) \xi\right]}{\left(-1+\mathrm{e}^{2(L a+L b) m}\right) p\left(-m^{2}+\xi^{2}+p^{2} \tau_{2}^{2}\right)} \\
\frac{\tilde{\bar{\phi}}}{-m^{2}+\xi^{2}+p^{2} \tau_{2}^{2}} \\
0
\end{array}\right]
\end{aligned}
$$




$$
\left\{\begin{aligned}
K_{1,1} & =\frac{1}{2 \pi} \int_{0}^{\infty} 2 \sum_{i=1}^{4}\left(\frac{-\mathrm{i} b_{i} \lambda_{i}}{\xi}-v\right) \mathbf{A}_{i, 7}^{-1} \cos \left[\xi\left(X_{j}-\eta_{k}\right)\right] \mathrm{d} \xi \\
K_{1,2} & =\frac{1}{2 \pi}\left\{\int_{0}^{\infty}\left[2 \sum_{i=1}^{4}\left(\frac{-b_{i} \lambda_{i}}{\xi}+\mathrm{i} v\right) \mathbf{A}_{i, 8}^{-1}-\frac{1-v^{2}}{2}\right] \sin \left[\xi\left(X_{j}-\eta_{k}\right)\right] \mathrm{d} \xi+\frac{1-v^{2}}{2} \frac{1}{X_{j}-\eta_{k}}\right\} \\
W_{1} & =\frac{1}{2 \pi} \int_{0}^{\infty}\left[\sum_{i=1}^{4} \sum_{j=1}^{8}\left(\mathrm{i} v \xi-b_{i} \lambda_{i}\right)\left(\mathbf{A}_{i j} \cdot \gamma_{j}\right)+\frac{\mathrm{i}\left(1-\mathrm{e}^{2 L a m}\right)\left(1+\mathrm{e}^{2 L b m}\right)\left((1-v) \xi^{2}+p^{2} \tau_{2}^{2}\right)}{2\left(-1+\mathrm{e}^{2(L a+L b) m}\right) \xi\left(-m^{2}+\xi^{2}+p^{2} \tau_{2}^{2}\right)}\right] \tilde{\bar{\phi}} \cos \left(\xi X_{j}\right) \mathrm{d} \xi \\
& +\frac{2 \pi \mathrm{e}^{L a p \tau_{2}}\left(1-\mathrm{e}^{2 L b p \tau_{2}}\right) \tau_{2}^{2}}{\left(1-\mathrm{e}^{2(L a+L b) p \tau_{2}}\right)\left(1+p \tau_{1}+p \tau_{2}^{2}\right)}+\frac{2 \pi \mathrm{e}^{L a m}\left(-1+\mathrm{e}^{2 L b m_{0}}\right) \tau_{2}^{2}}{\left(1-\mathrm{e}^{2(L a+L b) m}\right)\left(1+p \tau_{1}+p \tau_{2}^{2}\right)} \\
K_{2,1} & =\frac{1}{2 \pi}\left[\int_{0}^{\infty}\left(2 \sum_{i=1}^{4} \frac{\mathrm{i} \frac{\xi}{\xi} b_{i}-\lambda_{i}}{\xi} \mathbf{A}_{i, 7}^{-1}-(1+v)\right) \operatorname{Sin}[\xi(x-\eta)] \mathrm{d} \xi+\frac{1+v}{X_{j}-\eta_{k}}\right] \\
K_{2,2} & =\frac{1}{2 \pi} \int_{0}^{\infty} 2 \sum_{i=1}^{4}\left(\frac{-\mathrm{i} \lambda_{i}}{\xi}-b_{i}\right) \mathbf{A}_{i, 8}^{-1} \cos \left[\xi\left(X_{j}-\eta_{k}\right)\right] \mathrm{d} \xi \\
W_{2} & =\frac{1}{2 \pi} \int_{0}^{\infty}\left[\sum_{i=1}^{4} \sum_{j=1}^{8}\left(\mathrm{i} \xi b_{i}-\lambda_{i}\right)\left(\mathbf{A}_{i, j}^{-1} \cdot \gamma_{j}\right)+\frac{\left(1+\mathrm{e}^{2 L a m}\right)\left(1+\mathrm{e}^{2 L b m}\right) m}{\left(-1+\mathrm{e}^{2(L a+L b) m}\right)\left(m^{2}-\xi^{2}-p^{2} \tau_{2}^{2}\right)}\right] \tilde{\bar{\phi}} \cdot(-\mathrm{i}) \operatorname{Sin}\left[\xi X_{j}\right] \mathrm{d} \xi
\end{aligned}\right.
$$

\section{References}

[1] Padture NP, Gell M, Jordan EH. Thermal barrier coatings for gas-turbine engine applications. Science 2002;296:280-4.

[2] Evans AG, Mumm DR, Hutchinson JW, Meier GH, Pettit FS. Mechanisms controlling the durability of thermal barrier coatings. Prog Mater Sci 2001;46:505-53.

[3] Evans AG, He MY, Hutchinson JW. Mechanics-based scaling laws for the durability of thermal barrier coatings. Prog Mater Sci 2001;46:249-71.

[4] Vassen R, Jarligo MO, Steinke T, Mack DE, Stover D. Overview on advanced thermal barrier coatings. Surf Coat Technol 2010;205:938-42.

[5] Mauer G, Vassen R. Current developments and challenges in thermal barrier coatings. Surface Engng 2011;27:477-9.

[6] Vaßen R, Kerkhoff G, Stover D. Development of a micromechanical life prediction model for plasma sprayed thermal barrier coatings. Mater Sci Engng: A 2001:303:100-9.

[7] Shao YF, Xu XH, Meng SH, Bai GH, Jiang CP, Song F. Crack patterns in ceramic plates after quenching. J Am Ceram Soc 2010;93:3006-8.

[8] Chen ZT, Hu KQ. Thermo-elastic analysis of a cracked half-plane under a thermal shock impact using the hyperbolic heat conduction theory. J Therm Stress 2012;35:342-62.

[9] Ali YM, Zhang LC. Relativistic heat conduction. Int J Heat Mass Trans 2005;48:2397-406.

[10] Keles I, Conker C. Transient hyperbolic heat conduction in thick-walled FGM cylinders and spheres with exponentially-varying properties. Eur J MechA/Solids 2011;30:449-55.

[11] Dai H-L, Rao Y-N. Dynamic thermoelastic behavior of a double-layered hollow cylinder with an FGM layer. J Therm Stress 2013;36:962-84.

[12] Jordan PM, Puri P. Revisiting the Danilovskaya problem. J Therm Stress 2006;29:865-78.

[13] Wang H, Ding H, Chen Y. Dynamic solution of a multilayered orthotropic piezoelectric hollow cylinder for axisymmetric plane strain problems. Int J Solids Struct 2005;42:85-102.

[14] Zhu D, Miller RA, Nagaraj BA, Bruce RW. Thermal conductivity of EB-PVD thermal barrier coatings evaluated by a steady-state laser heat flux technique. Surf Coat Technol 2001;138:1-8.

[15] Martynyak R, Honchar KI, Nahalka S. Simulation of thermomechanical closure of an initially open interface crack with heat resistance. Mater Sci+ 2003:672-81.

[16] Hasselman D. Effect of cracks on thermal conductivity. J Compos Mater 1978;12:403-7.

[17] Kaminski W. Hyperbolic heat conduction equation for materials with a nonhomogeneous inner structure. J Heat Trans 1990;112:555-60.

[18] Graßmann A, Peters F. Experimental investigation of heat conduction in wet sand. Heat Mass Transfer 1999;35:289-94.

[19] Roetzel W, Putra N, Das SK. Experiment and analysis for non-Fourier conduction in materials with non-homogeneous inner structure. Int J Therm Sci 2003;42:541-52.

[20] Miller MK, Guy JWT. Numerical inversion of the Laplace transform by use of Jacobi polynomials. Siam J Numer Anal 1966;3:624-35.

[21] Chen Z. Interfacial coplanar cracks in piezoelectric bi-material systems under pure mechanical impact loading. Int J Solids Struct 2006;43:5085-99.

[22] Hu K, Chen Z. Thermoelastic analysis of a partially insulated crack in a strip under thermal impact loading using the hyperbolic heat conduction theory. Int J Engng Sci 2012;51:144-60.

[23] Brown E, Erdogan F. Thermal stresses in bonded materials containing cuts on the interface. Int J Engng Sci 1968;6:517-29.

[24] Delale F, Erdogan F. Effect of transverse shear and material orthotropy in a cracked spherical cap. Int J Solids Struct 1979;15:907-26.

[25] Theocaris P, Ioakimidis N. Numerical integration methods for the solution of singular integral equations (for crack tip stress intensity factor evaluation in elastic media). Q Appl Math 1977;35:173-83.

[26] Erdogan F, Gupta GD, Cook T. Numerical solution of singular integral equations. Methods of analysis and solutions of crack problems. Springer; 1973. p. $368-425$.

[27] Li W, Li J, Abdelmoula R, Song F, Jiang CP. Inertia effect analysis of a half-plane with an induced crack under thermal loading using hyperbolic heat conduction. ZAMM-J Appl Math Mech 2015. http://dx.doi.org/10.1002/zamm.201500133.

[28] Guo S, Wang B. Thermal shock fracture of a cylinder with a penny-shaped crack based on hyperbolic heat conduction. Int J Heat Mass Trans 2015;91:235-45.

[29] Padture NP, Gell M, Jordan EH. Thermal barrier coatings for gas-turbine engine applications. Science 2002;296:280-4.

[30] Choi Y, Jeon S, Jeon M-s, Shin H-G, Chun HH, Lee Y-s, et al. Crack propagation behavior of TiN coatings by laser thermal shock experiments. Appl Surf Sci $2012 ; 258: 8752-7$.

[31] Antaki PJ. New interpretation of non-Fourier heat conduction in processed meat. Trans ASME-C-J Heat Transfer 2005;127:189-93. 